\title{
Global Warming and Changes in Ocean Circulation
}

Philip B. Duffy

Ken G. Caldeira

February 1998

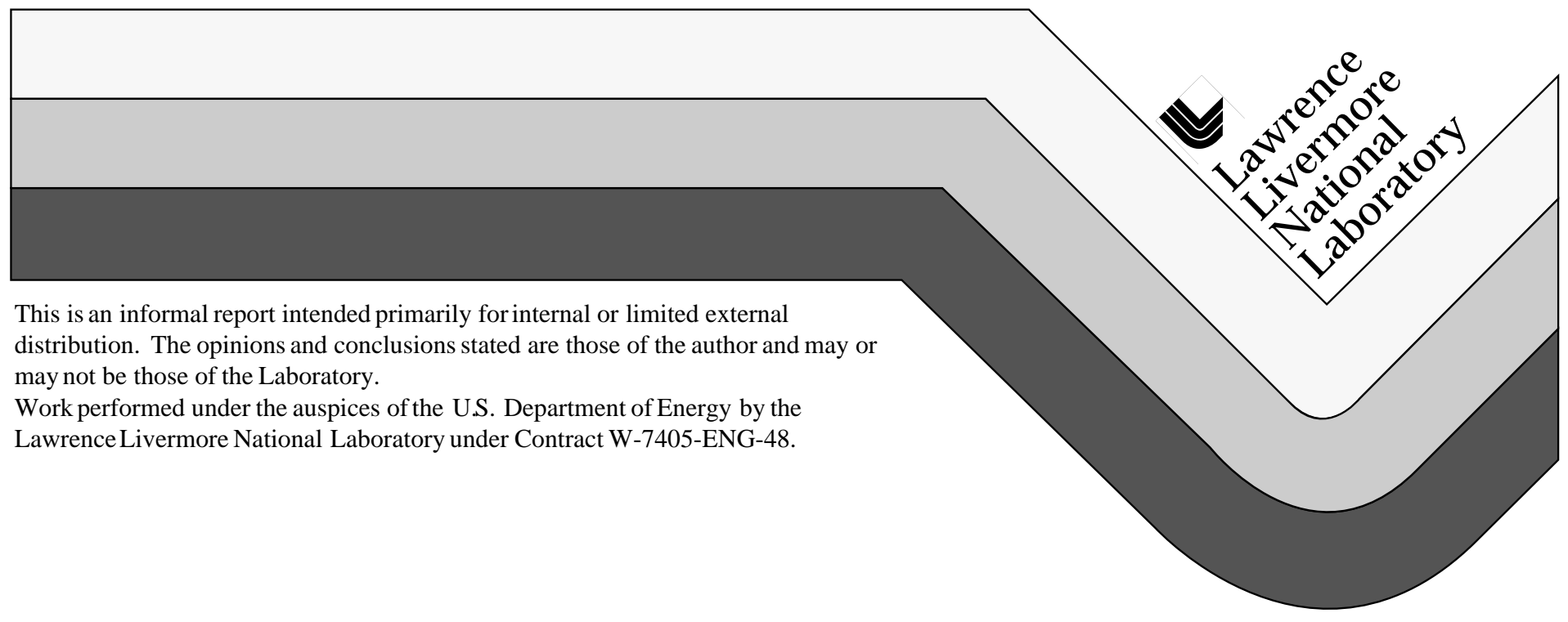




\section{DISCLAIMER}

This document was prepared as an account of work sponsored by an agency of the United States Government. Neither the United States Government nor the University of California nor any of their employees, makes any warranty, express or implied, or assumes any legal liability or responsibility for the accuracy, completeness, or usefulness of any information, apparatus, product, or process disclosed, or represents that its use would not infringe privately owned rights. Reference herein to any specific commercial product, process, or service by trade name, trademark, manufacturer, or otherwise, does not necessarily constitute or imply its endorsement, recommendation, or favoring by the United States Government or the University of California. The views and opinions of authors expressed herein do not necessarily state or reflect those of the United States Government or the University of California, and shall not be used for advertising or product endorsement purposes.

This report has been reproduced directly from the best available copy.

Available to DOE and DOE contractors from the Office of Scientific and Technical Information P.O. Box 62, Oak Ridge, TN 37831

Prices available from (423) 576-8401

Available to the public from the National Technical Information Service

U.S. Department of Commerce 5285 Port Royal Rd. Springfield, VA 22161 


\section{Global Warming and Changes in Ocean Circulation}

Tracking Code: 95-ERD-030

UCRL-ID-129587

Principal Investigator: Philip B. Duffy

Atmospheric Science Division

L-103

(510) 422-3722

pduffy@1lnl.gov

Co-Investigator: Kenneth Caldeira

Atmospheric Science Division

L-103

(510) 423-4191

kenc@llnl.gov

\section{Summary}

Recent modeling and observational work has raised the possibility that global warming might cause changes in the circulation of the ocean. Several distinct types of changes are conceivable. First, the warming of the surface ocean due to increased greenhouse warming ("global warming") would tend to reduce vertical density gradients, and thus reduce smallscale vertical turbulent mixing. Second, increased high-latitude precipitation associated with global warming, by reducing the density of surface water in the North Atlantic, could cause a slowdown or shutoff of the global thermohaline circulation. Finally, loss of Antarctic sea ice due to global warming could affect the circulation of the Southern Ocean and possibly other regions.

If such changes really occur, they could have important climatic consequences. For example, any slowdown of ocean circulation (of either the large-scale thermohaline circulation or small-scale turbulent mixing) would accelerate global greenhouse warming by reducing the amount of heat and fossil-fuel $\mathrm{CO}_{2}$ that the oceans absorb from the atmosphere. This absorption is currently a major mitigating factor in the rate of greenhouse 
warming; thus a slowing of ocean circulation due to greenhouse warming could act to accelerate that warming. In addition, these circulation changes could have significant effects on organisms living in the surface ocean, due to a reduced supply of nutrients. Finally, loss of sea ice would increase the ocean volume, leading to sea level rise, and would have unknown effects upon ocean circulation.

One technical goal of this project was to investigate some of these possible changes in ocean circulation in a quantitative way, using a state-of-the art numerical model of the ocean. Another technical goal was to develop our ocean model. This model is a detailed, three-dimensional numerical model of the ocean circulation and ocean carbon cycle. In this model, the variables involving ocean circulation (e.g., temperature, salinity, and velocities) and the biogeochemical variables (e.g., ${ }^{12} \mathrm{C},{ }^{13} \mathrm{C},{ }^{14} \mathrm{C}$ ) are defined on a three-dimensional grid that describes latitude, longitude, and depth. In terms of the physics, biology, and chemistry it includes, this model is possibly the most advanced of its kind in the world. In addition, our model is computationally state-of-the-art, in that it runs with good speed and efficiency on the latest, massively parallel, computers (e.g. Cray T3D, IBM SP-2).

A major non-technical goal of the project was to establish LLNL as a center of excellence in modeling of the ocean circulation and carbon cycle, by producing high-quality peerreviewed publications and conference talks.

In the early years of this project, we focused on the development of the ocean circulation/ocean biogeochemistry model. As part of this process, we performed a number of important studies to evaluate various ways to represent "subgrid scale" motions in ocean models - those motions whose spatial scale is smaller than individual model grid cells and are therefore not resolved by the model. Good representations of subgrid scale 
motions are needed for many reasons; one that has particular relevance to this project is to represent the reduced vertical turbulent mixing, discussed above, that may accompany global warming.

As part of this evaluation of competing representations of subgrid scale motions, we showed that more advanced representations of subgrid scale motions cause an unexpectedly large reduction in calculated uptake by the ocean of anthropogenic $\mathrm{CO}_{2}$. Because this uptake directly influences atmospheric $\mathrm{CO}_{2}$ levels, a reduction in ocean uptake of anthropogenic $\mathrm{CO}_{2}$ implies more rapid rates of predicted greenhouse warming.

A major result of this project was the finding that certain interactions between the ocean and sea ice have important and unexpected effects on the results of our ocean model (and, by implication, on the real ocean). We found that more careful modeling of interactions between the ocean and sea ice (specifically, how salt rejected during formation of ice is handled) eliminated several important problems which have always plagued numerical ocean models. These problems, poor simulation of salinity and of uptake of "transient tracers"- substances which enter the ocean over a limited period of time-are illustrated below; also shown are the improved results obtained with our improved model treatment of ocean-ice interactions.

These results suggest that sea ice-especially in the Southern Hemisphere-has an important role in maintaining the vertical density structure and circulation of the ocean. The mechanism is as follows: formation of sea ice, which contains very little salt, results in salt rejection and an increase in the salinity of local surface water. This salty, dense water tends to sink, increasing the salinity and density of subsurface water. Melting of sea ice places fresh water on the ocean surface and thus reduces the density of the surface water. 
Consequently, the seasonal cycle of freezing and melting of sea ice decreases the density of surface water and increases the density of subsurface water; i.e. increases vertical density gradients in the upper ocean. Thus, we showed that sea ice plays an important and unappreciated role in maintaining the density and circulation of the Southern Ocean. This suggests that loss of Antarctic sea ice, such as through global warming, would have important and unexpected effects on the large-scale ocean circulation, and hence on global and regional climate.

Finally, our work on modeling of ocean/sea-ice interactions lead to the finding-paid for under other funding - that ocean models traditionally overestimate uptake of transient tracers. This result has very important implications. Simulations of ocean uptake of anthropogenic $\mathrm{CO} 2$ - a very important transient tracer — cannot be verified directly against observations because one cannot observationally distinguish anthropogenic carbon from natural carbon in the ocean. Thus these simulations must be tested indirectly. Perhaps the best such test is to simulate ocean uptake of chloroflourocarbons (CFCs). Concentrations of CFCs in the ocean correlate very strongly (at least in models) with correlations of anthropogenic $\mathrm{CO}$. Thus a model that accurately simulates ocean uptake of CFCs is more likely to accurately simulate uptake of anthropogenic $\mathrm{CO} 2$ than is a model which represents CFC uptake poorly. Our work here on improving the simulation of ice-ocean interactions focused our attention on the fact that most ocean models grossly overestimate the fluxes of CFC into the Southern Ocean. This strongly suggests that simulated fluxes of anthropogenic $\mathrm{CO} 2$ into the Southern Ocean—which are believed to be higher than fluxes elsewhere-are also overestimated. Thus work performed under this project has lead us to the conclusion that the conventional wisdom—which holds that fluxes of anthropogenic $\mathrm{CO} 2$ into the Southern Ocean are much higher than fluxes elsewhere-is almost certainly 
completely erroneous, being based on poor simulations of ocean circulation. This conclusion is important because, based on these poor model simulations, significant resources are being devoted to observing carbon isotopes and other quantities in the Southern Ocean.

\section{Problem Definition}

This work investigated one of the most serious and poorly understood possible effects of anthropogenic climate change: possible changes in ocean circulation. As discussed below, several types of ocean circulation changes might be triggered by human activities. Neither the likelihood of these circulation changes, nor their effects, are well understood.

\section{Background}

Recent modeling and observational work has raised the possibility that global warming might cause changes in the circulation of the ocean. Several distinct types of changes are conceivable. First, the warming of the surface ocean due to increased greenhouse warming ("global warming") would tend to reduce vertical density gradients, and thus reduce vertical turbulent mixing. This type of mixing occurs on very local spatial scales. Second, as discussed in detail below, increased high-latitude precipitation associated with global warming, by reducing the density of surface water in the North Atlantic, could cause a slowdown or shutoff of the global thermohaline circulation. This would have a significant effect on regional climates, since it would result in a significant redistribution of heat around the globe. Finally, loss of Antarctic sea ice due to global warming could affect the circulation of the Southern Ocean. Observations (W.K. de la Mare, Abrupt mid-twentiethcentury decline in Antarctic sea-ice extent from whaling records, Nature, 389, 57-59, 1997) 
suggest that the areal extent of Antarctic sea ice diminished by about $25 \%$ between the 1950s and 1970s.

As mentioned above, a slowdown in the large-scale thermohaline circulation might have important climatic consequences. The other types of possible ocean circulation changes discussed above (a reduction in small-scale mixing and a reduction in sea ice extent and thickness) might also have significant effects. Reductions in small-scale vertical mixing could have significant effects on organisms living in the surface ocean, due to a reduced supply of nutrients. Reduced vertical mixing would also reduce the amount of $\mathrm{CO}_{2}$ and heat absorbed by the ocean, thus accelerating greenhouse warming. Loss of sea ice would increase the ocean volume, leading to sea level rise, and would have unknown effects upon ocean circulation.

\section{Objectives:}

Besides the technical goals listed below, this project had the institutional goal of establishing LLNL as a center of excellence in the field of ocean-climate modeling. As discussed below, we were highly successful in meeting this goal. The technical goals of this project were to:

(1) develop state of the art models of ocean circulation and ocean biogeochemistry. In addition to being state of the art scientifically, we wanted our models to be capable of running on the latest massively parallel computers (i.e. to be state of the art computationally as well).

(2) investigate better representations in ocean models of the effects of subgrid scale ocean circulation (i.e. of circulation not resolved by ocean models). We investigated improved 
model treatments of several types of subgrid scale motions: lateral transport by eddies, vertical turbulent mixing, and convection. In particular, we wanted to test various representations of subgrid scale motions by seeing which of them allow the most accurate simulation of carbon isotopes and related quantities. We selected this emphasis because these quantities cannot be simulated by most other ocean modeling groups. Thus this avenue of research exploits one of our unique capabilities.

(3) demonstrate our ocean modeling capabilities by performing coupled ocean circulation/ocean carbon cycle calculations (e.g. we calculated the uptake by the ocean of anthropogenic — essentially fossil-fuel- $\mathrm{CO}_{2}$.)

(4) investigate possible changes in ocean circulation that might be caused by human activities;

(5) develop an improved dataset of river runoff (i.e. river discharges in to the ocean). These data can be used as inputs to numerical ocean models, since river discharges constitute part of the fresh water "forcing" at the ocean surface.

(6) investigate and develop methods for speeding up ocean model calculations for the important class of problems in which the ocean circulation is not expected to change from year to year.

\section{Results}

Model development We completed development of our ocean biogeochemistry model (OBGCM), and coupled it to our ocean circulation model. In this coupled model, physical transport of biogeochemical tracers is accomplished by the ocean circulation model; air-sea 
gas exchange, chemical reactions, and biological transport of carbon isotopes are accomplished by the OBGCM.

We developed a simple, three-dimensional diffusive ocean model and coupled it to our ocean biogeochemistry model. The results of this simple coupled model were used to debug and set parameter values in the ocean biogeochemistry model, without the expense associated with coupling it to the full ocean circulation model.

We did several substantial calculations using the full coupled ocean circulation/biogeochemistry model . These include simulations of (a) the pre-industrial ocean; (b) today's ocean; (c) ocean uptake of bomb ${ }^{14} \mathrm{C}$; (d) ocean uptake of anthropogenic carbon from the atmosphere. We also evaluated the effects of different representations of subgrid scale motions on simulated concentrations of carbon isotopes, etc.

Model evaluation: We developed an innovative approach to measuring the realism of the simulated circulation in our OGCM. There are no sources or sinks of heat or salinity in the subsurface ocean, yet spurious heat and salinity sources and sinks would be required to maintain observed temperature and salinity fields in the OGCM. A reduction in these spurious sources and sinks indicates improvements in modeled ocean circulation. We calculated the required spurious sources and sinks and are now analyzing the results. Our results indicate that the Gent-McWilliams parameterization markedly improves ocean circulation in the Southern Ocean and in the North Atlantic Ocean.

Improved representations of subgrid scale motions (eddies and vertical mixing): ) In collaboration with J. C. McWilliams and colleagues, we implemented in our ocean 
circulation model an improved sub grid scale mixing parameterization (the "GentMcWilliams" parameterization). This parameterization has been shown to greatly improve the simulated temperature structure in models like ours; we showed that it does so without significantly changing the already good simulation of uptake of transient tracers. This means that the ability of the model to simulate the absorption of transient tracers (notably anthropogenic $\mathrm{CO}_{2}$, the primary cause of enhanced greenhouse warming) while at the same time properly simulating the ocean's temperature structure is greatly improved. Two peer-reviewed papers by P. Duffy et al. describing this and other effects of this parameterization were published in 1995 (see publication list below). In collaboration with Prof. M. I. Hoffert of New York University, we implemented in our ocean circulation model an improved parameterization of vertical transport of tracers by subgrid scale eddies. (This is complementary to the Gent-McWilliams parameterization discussed above, which deals with lateral transport.) This parameterization allows our model to reflect the fact that vertical transport by subgrid scale eddies will change (relative to today's ocean) as the ocean density structure changes due to greenhouse warming. A paper describing this work was published in J. Phys. Oceanogr.

Improved representations of subgrid scale motions (convection): We performed sensitivity studies with our ocean circulation model which demonstrated that the results improve dramatically when a crude representation of subgrid scale convection beneath sea ice is added to the model. In particular, simulated salinities and simulated uptake of chloroflourocarbons (CFCs) improved dramatically.

The previous inability to accurately simulate these two quantities was a very serious problem. Accurate representation of salinity is critical in ocean models, because salinity 
in part determines the density of seawater; the density in turn helps determine the circulation. Thus one cannot hope to accurately simulate ocean circulation without a good representation of salinity. Until our work here, ocean models had nearly always produced very unrealistic simulations of salinity.

Simulated uptake of CFCs is a very important test of ocean models because it is a surrogate for simulated uptake of anthropogenic carbon. (Simulating ocean uptake of anthropogenic carbon is critical because it is directly related to predicting future atmospheric concentrations of $\mathrm{CO}_{2}$, and thus future climate.) Because anthropogenic carbon in the ocean cannot be distinguished observationally from natural carbon, one cannot directly verify simulations of ocean uptake of anthropogenic carbon against observations. Instead, indirect tests must be used. Perhaps the most important of these is ocean uptake of CFCs, because (at least in models) concentrations of CFCs in the ocean correlate strongly with concentrations of anthropogenic $\mathrm{CO}_{2}$. Thus, a realistic simulation of ocean CFC uptake increases confidence in that model's ability to simulate uptake of anthropogenic $\mathrm{CO}_{2}$. Conversely, an unrealistic simulation of ocean $\mathrm{CFC}$ uptake suggests that simulated uptake of anthropogenic $\mathrm{CO}_{2}$ will also be unrealistic.

Until recent work by us, which was paid for by other funding but made possible by this project, ocean model simulations of CFC uptake were nearly all very unrealistic in the Southern Ocean: simulated uptake was much too high. This strongly implies that these models were also grossly overpredicting ocean uptake of anthropogenic $\mathrm{CO}_{2}$ in this region. Our results suggest that the conventional wisdom, which holds that fluxes of anthropogenic $\mathrm{CO}_{2}$ into the Southern Ocean are much higher than elsewhere, is completely wrong, and is an artifact of poor modeled ocean circulation in this region. This is an important result, because, on the basis of these probably erroneous model 
simulations, millions of dollars are now being spent making observations of carbon isotopes and related quantities in the Southern Ocean.

To summarize this work, by making a small change in our model, we were able to eliminate two serious problems which have plagued ocean models for years or decades: poor representations of salinity and poor representations of transient tracer uptake. In addition, our results strongly suggest that the widespread point of view that fluxes of anthropogenic $\mathrm{CO}_{2}$ into the Southern Ocean are higher than elsewhere is wrong, because it is based on poor simulations of ocean circulation.

Ocean model speedup: We used the technique known as "time step splitting" to perform the simulations needed for this project. This reduced the run-time of our ocean general circulation model by a factor of 24 compared to the standard modus operandi. In addition, we have conceived an innovative approach to developing the separate tracer transport model described above. This approach will make use of both the speed and the copious memory of the Cray T3D massively parallel computer; we estimate that this transport model will speed up ocean calculations by a factor of 2 to 10 relative to the time-step splitting technique; i.e., by a factor of 48 to 240 compared to the standard model run in the standard mode. N.B.: both of these approaches to speeding up the ocean calculation can be used only in problems where the ocean circulation does not vary from year to year. Thus they can not be used for climate change simulations, where the ocean circulation might be expected to change.

Observed river dataset: One of the input quantities required by numerical ocean models is the flux of fresh water (due to precipitation, evaporation, etc.) at the ocean surface. Part of this flux comes in the form of river discharges. Thus, a relatively complete dataset of observed river discharges is useful for running numerical ocean models. Until our recent 
work, the largest published dataset of this type included only about 50 rivers, and only about half the expected discharge into the ocean To remedy this inadequacy, we compiled the most extensive dataset ever assembled of river discharges to the ocean. Our dataset includes nearly 1,000 rivers, which account for roughly $70 \%$ of the discharge into the ocean. (The other $30 \%$ is mostly in the form of tiny, unmeasured rivers.) Our accomplishment was to compile and apply quality control to as many published river discharge measurements as we could find. A paper describing this dataset was published in J. Geophys. Res.. In addition, the dataset is available electronically over the internet, and has been requested and used by many researchers world-wide.

In addition to compiling river discharge measurements, we also performed new and important analyses of the global fresh-water budget. As discussed above, our dataset of approximately 1,000 rivers accounts for about $70 \%$ of the total discharge into the ocean (as estimated independently, from measurements of precipitation and evaporation over land). We showed that the size distribution of river discharges is log-normal for three orders of magnitude of river sizes. If we extrapolate that size distribution to include smaller rivers (which are too small and too numerous to include individually in our dataset) we find that the total estimated river flow for all rivers exactly matches that estimated independently, from measurements of precipitation and evaporation over land. Thus, our analysis presents, for the first time, a self-consistent picture of the global fresh-water budget.

\section{Conclusions}

This project had significant scientific and institutional impact. Scientifically, we achieved a number of important results. The most important is the finding that adding a simple representation of subgrid scale convection to our ocean model eliminated two serious problems that have plagued ocean models for years or decades. (These problems are poor 
representation of salinity and poor representation of uptake of transient tracers in the Southern Ocean.) This lead us to the conclusion that the conventional wisdom-which holds that fluxes of anthropogenic carbon into the Southern Ocean are much higher than fluxes into other parts of the ocean-is probably completely erroneous, since it is based on faulty model simulations. This is a significant finding, since, on the basis of these faulty model simulations, significant resources are now being devoted to making observations of carbon isotopes and related quantities in the Southern Ocean.

In terms of institutional impact, this project was very effective at establishing LLNL as a center of excellence in modeling the ocean circulation and ocean carbon cycle. We are now one of only three institutions in the US (the other two are NCAR and GFDL/Princeton) which have the capability to do coupled ocean circulation/carbon cycle simulations. The ability to perform these simulations is critical to predicting future climate, since they are required in order to predict ocean uptake of anthropogenic $\mathrm{CO}_{2}$ (which in part determines how much $\mathrm{CO}_{2}$ remains in the atmosphere). Among these three institutions, we are unique in that our models run on massively parallel computers. In short, our ocean models are state of the art scientifically and computationally, and this fact is widely recognized in the climate research community.

\section{Refereed Publications}

Duffy, P.B., D. Eliason, A. J. Bourgeois, C. Covey (1995), "Simulation of Bomb Radiocarbon in Two Ocean General Circulation Models," Geophysical Res. 100, 22,545565 (UCRL-JC-115622).

Duffy, P.B., P. Eltgroth, A. J. Bourgeois, K. Caldeira (1995), “Effect of Improved Subgrid Scale Transport of Tracers on Uptake of Bomb Radiocarbon in the GFDL Ocean 
General Circulation Model," Geophys. Research Lett., 22, 1065-1068 (UCRL-JC119137).

Perry, G., P. B. Duffy, N. Miller (1996), “An Extended Dataset of River Discharges for Validation of Climate Models," J. Geophys. Res., 101, 21, 339-34 (UCRL-JC-121799).

Duffy, P.B., K. Caldeira, J. Selvaggi, M. Hoffert, "Effect of Subgrid Scale Mixing Parameterizations of Simulated Distributions of Natural ${ }^{14} \mathrm{C}$, Temperature, and Salinity in a Three-Dimensional Ocean General Circulation Model,” J. Phys. Oceanogr., 27, 498-523 1997 (UCRL-JC-125024).

Duffy, P., and K. Caldeira (1997), "Sensitivity of Simulated Salinity in a Threedimensional Ocean Model to Upper-Ocean Transport of Salt from Seal-Ice Formation,” Geophys. Res. Lett, 24, 1323-1326 (UCRL-JC-125185).

Caldeira, K., and P. Duffy (1997), "Sensitivity of simulated CFC-11 distributions in a global ocean model to the treatment of salt rejected during sea-ice formation," Geophys. Res. Lett. , in press. (UCRL-JC-127053).

\section{Meeting Abstracts}

Duffy, P., D. Eliason, A. Bourgeois, "Simulation of Bomb Radiocarbon in Two Global Ocean General Circulation Models, Part 1," American Geophysical Union/American Society of Limnology \& Oceanography Ocean Sciences Meeting Feb. 21-25, 1994, San Diego, CA (UCRL-JC-115622).

Duffy, P., K. Caldeira, J. Selvaggi, M. Hoffert (1996), "Effect of Subgrid Scale Eddy Parameterizations on Simulation of Natural ${ }^{14} \mathrm{C}$ in a three-dimensional Ocean GCM,” 
American Geophysical Union Fall Meeting, San Francisco, CA 1995 (UCRL-JC-122219ABS).

Perry, G., P. B. Duffy, N. Miller (1996), “An Extended Dataset of River Discharges for Validation of Climate Models," American Geophysical Union Fall Meeting, San Francisco, CA 1995 (UCRL-JC-121799-ABS).

Duffy, P., and K. Caldeira, "Effect of the Gent-McWilliams Tracer Transport Parameterization on the Simulated Distribution of Natural ${ }^{14} \mathrm{C}$ in the Ocean," American Geophysical Union/American Society of Limnology \& Oceanography Ocean Sciences Meeting, Feb. 21-25, 1994, San Diego, CA (UCRL-JC-122219-ABS).

Wickett, M., P. Duffy, K. Caldeira (1996), "Effect of Mediterranean Outflow on the Global Thermohaline Circulation," American Geophysical Union/American Society of Limnology and Oceanography Ocean Sciences Meeting, Feb. 1996, San Diego, CA (UCRL-JC-122220-ABS).

Eliason, D., P. Duffy, A. Bourgeois (1996), "Simulation of Bomb Radiocarbon in Two Global Ocean General Circulation Models, Part 2: Comparison to Data,” American Geophysical Union/American Society of Limnology \& Oceanography Ocean Sciences Meeting, Feb. 21-25, 1994, San Diego, CA (UCRL-JC-115622). 


\section{Figures}

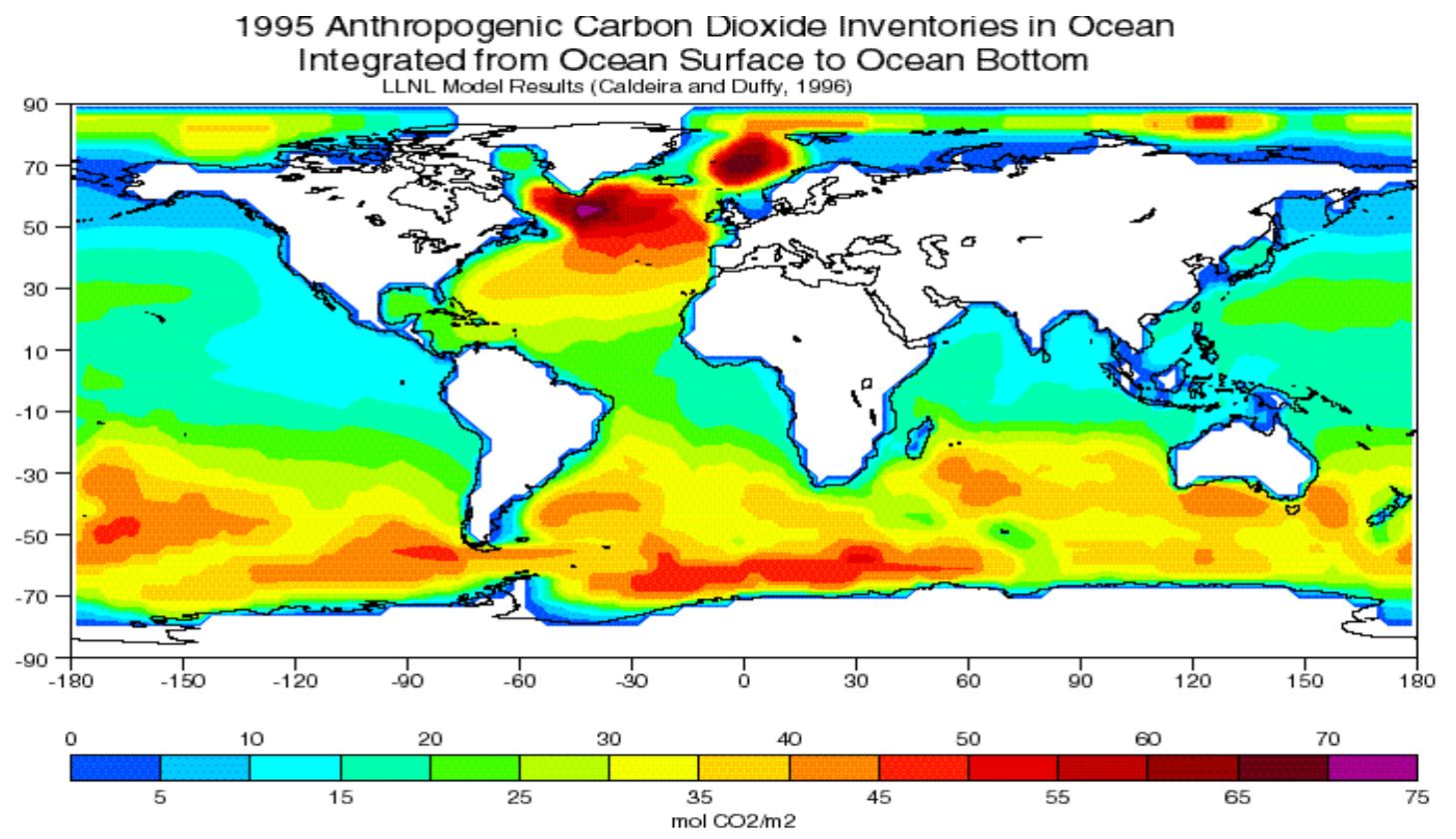

Figure 1: Simulation of the column inventory (vertical integral of the concentration) of anthropogenic $\mathrm{CO}_{2}$ in the ocean as of 1995. This (essentially) is fossil fuel carbon which has been removed form the atmosphere. This simulation shows the conventional result, which is that column inventories of anthropogenic $\mathrm{CO}_{2}$ are much higher in the Southern Ocean than elsewhere. As discussed in the text, our results strongly suggest that this conventional result is wrong, and that column inventories of anthropogenic $\mathrm{CO}_{2}$ in the Southern Ocean are actually not much higher than elsewhere. 


\section{Atlantic Ocean Salinity}
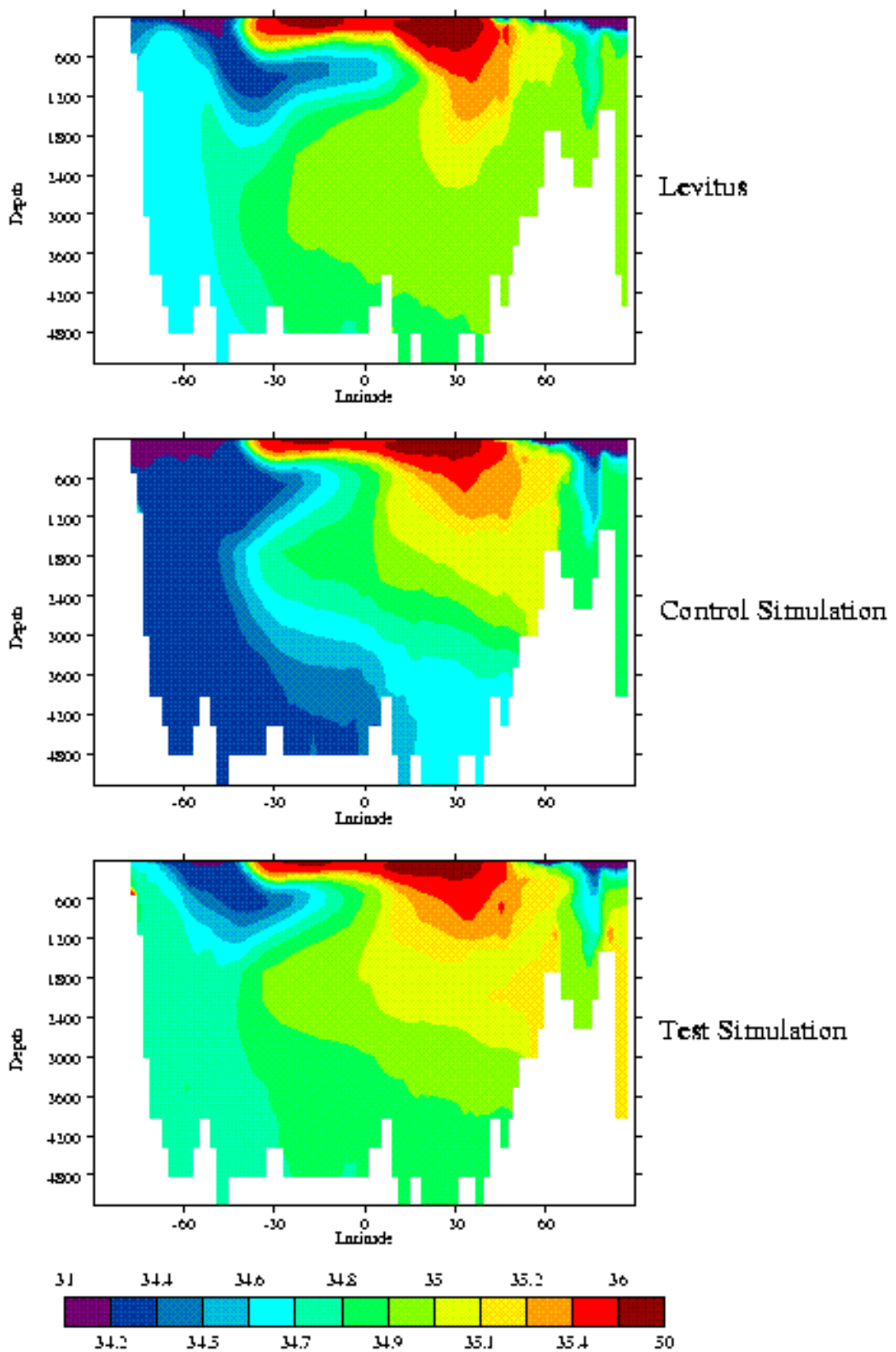

Figure 2: observations (top panel) and two simulations of salinity in the Atlantic ocean. The middle panel, which is typical of results produced with numerical ocean models, show unrealistically low salinities in much of the deep ocean. The simulation shown in the bottom panel, which uses a crude treatment of subgrid scale convection developed by us, has much more realistic salinities. Poor representation of salinity is a serious problem which, until now, has plagued ocean models. 


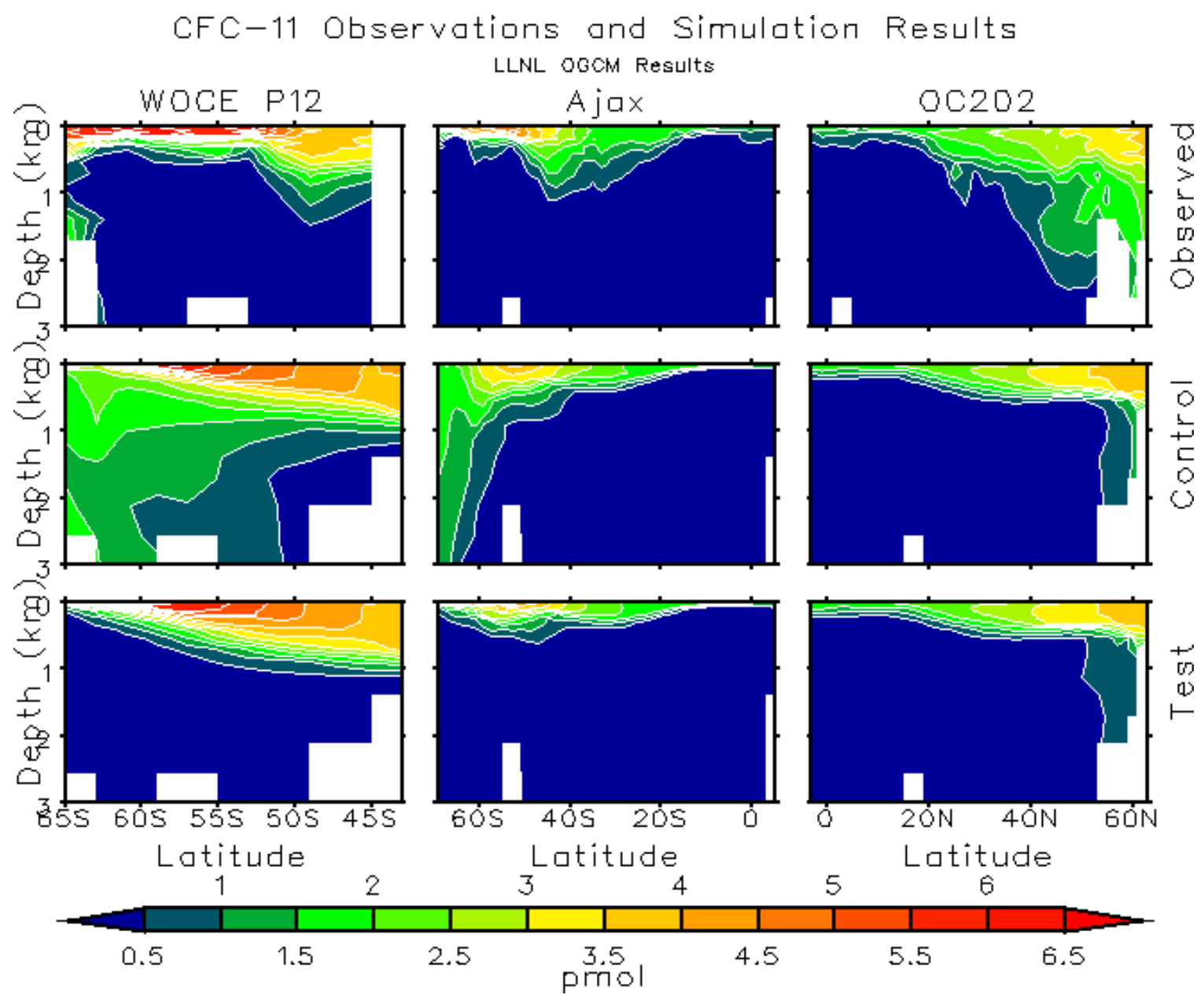

Figure 3: observations (top row) and two simulations of CFCs in the ocean. Each panel shows a latitude (horizontal axis) vs. depth (vertical axis) section. The left-hand column represents a longitude slice in the Southern Ocean; the middle column shows the South Atlantic Ocean; the right column shows the North Atlantic. The middle row, which shows a simulation which is typical of results produced with numerical ocean models, show unrealistically high CFC concentrations in the Southern Ocean and South Atlantic (left and middle columns). The simulation shown in the bottom row, which uses a crude treatment of subgrid scale convection developed by us, has much more realistic CFC concentrations. Poor representation of CFCs is a serious problem, because it strongly suggests that the same models are over-predicting uptake by the ocean of anthropogenic $\mathrm{CO}_{2}$ and thus underpredicting rates of future greenhouse warming. 


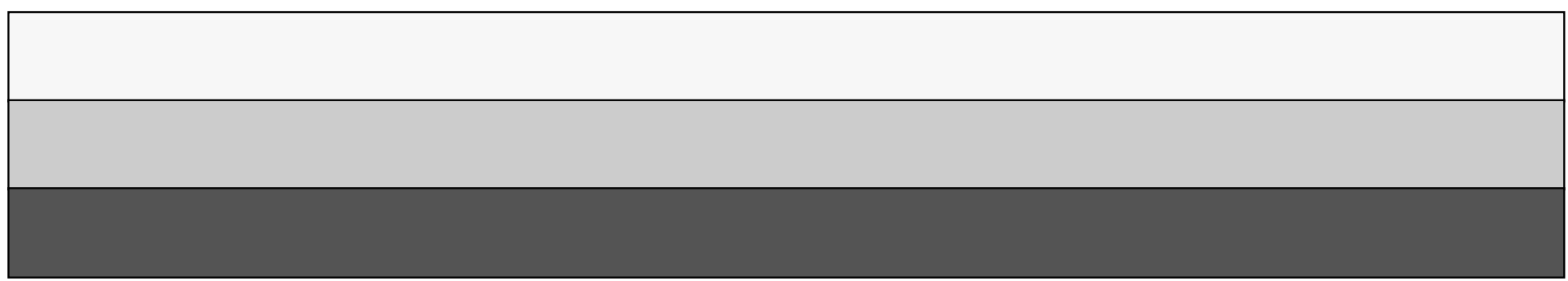

